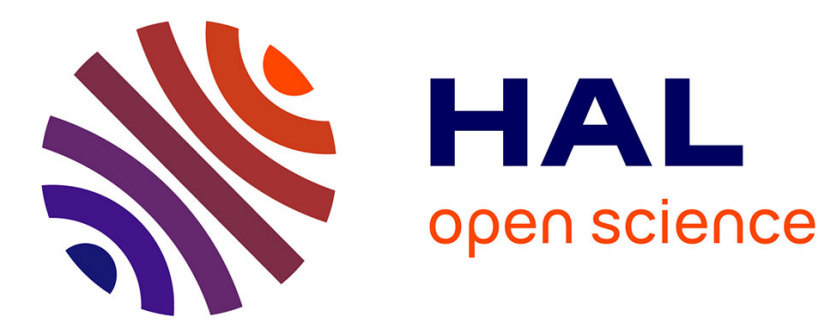

\title{
The study of thermal processes in an electrode submitted to an electric arc
}

\author{
J. Devautour, J. Chabrerie, Ph. Testé
}

\section{To cite this version:}

J. Devautour, J. Chabrerie, Ph. Testé. The study of thermal processes in an electrode submitted to an electric arc. Journal de Physique III, 1993, 3 (6), pp.1157-1166. 10.1051/jp3:1993191 . jpa-00248989

\section{HAL Id: jpa-00248989 https://hal.science/jpa-00248989}

Submitted on 1 Jan 1993

HAL is a multi-disciplinary open access archive for the deposit and dissemination of scientific research documents, whether they are published or not. The documents may come from teaching and research institutions in France or abroad, or from public or private research centers.
L'archive ouverte pluridisciplinaire HAL, est destinée au dépôt et à la diffusion de documents scientifiques de niveau recherche, publiés ou non, émanant des établissements d'enseignement et de recherche français ou étrangers, des laboratoires publics ou privés. 
Classification

Physics Abstracts

$02.70-06.70 \mathrm{E}-44.10-52.80 \mathrm{M}$

\title{
The study of thermal processes in an electrode submitted to an electric arc
}

\author{
J. Devautour ( $\left.{ }^{1}\right)$, J. P. Chabrerie $\left({ }^{2}\right)$ and $\mathrm{Ph}$. Testé $\left({ }^{3}\right)$ \\ (1) L.G.E.P. \& Comptoir Lyon-Alemand-Louyot, 8 rue Portefoin, 75139 Paris, France \\ (2) Laboratoire de Génie Electrique de Paris. CNRS (ÜRA 127), Ecole Supérieure d'Electricité, \\ Plateau du Moulon, 91192 Gif sur Yvette, France \\ ( $\left.{ }^{3}\right)$ LGEP \& Electricité de France Les Renardières, 77250 Moret sur Loing, France
}

(Received 4 June 1992, revised 8 October 1992, accepted 3 Nov'ember 1992)

\begin{abstract}
This article investigates the thermal phenomena that are occurring in an electrode submitted to an electric arc in air at atmospheric pressure. Our purpose is to get a better understanding of the electrode erosion phenomena concerning vaporization or liquid droplets ejection. For that we propose an original experimental device allowing the measurement of the liquid and vapor quantities created under the arc root. The principle of this device is based on the ejection of the liquid metal through centrifugal movement of the electrodes during the arc. Some results are given relatively to $\mathrm{Ag}, \mathrm{Cu}$ and $\mathrm{AgSnO}_{2}$ cathodes and anodes. Then we present a numerical modelling of the thermal phenomena induced in the electrode by the heat transferred from the arc root. We propose a method to solve the difficulties related to this kind of $*$ free boundary » problems induced by the fact that the electrode material may undergo various phase changes. Finally we show how to use this numerical computation and the experimental results in order to estimate the power density input at a copper anode.
\end{abstract}

\section{Introduction.}

The generation of an electric arc between electrodes remains a widespread means of interrupting an alternative or direct current. In such a process, electrode materials are submitted to the intense surface heating of the arc root that brings a huge power density to it and causes subsequent erosion :

- by vaporization ;

- by liquid metal departure under the action of the different body or surface forces exerted on the liquid pool;

- by micro-explosions.

The aim of this study is to get a better understanding of the thermal phenomena occurring in an electrode. Indeed, before apprehending the complex mechanisms that govern erosion, it is 
primordial to determine quantitatively the amount of vapor and liquid created under the arc root. In a first part we'll describe an experimental device that permits to measure them. In a second part a $2 \mathrm{D}$ thermal model will be presented that allows to calculate these quantities. As phase changes may occur we propose an original solution of the corresponding free boundary problem.

An experimental set up permitting to evaluate the vapor and liquid quantities created by an arc.

DESCRIPTION. - Figure 1 and figure 2 are respectively giving a mechanical and an electrical schemes of the experimental device.

Two electrodes (1) are fixed, a distance $d$ apart, in a cylindrical vessel (2) along a radial direction. This vessel is made of a conducting cylinder and possesses a vertical shaft (3), fixed at its bottom and electrically insulated from it by means of a glass-epoxy piece (4). Ballbearings (5) allow this vessel to be rotated at an arbitrary angular frequency $\omega$ up to 300 rad.s ${ }^{-1}$ As we can see in figure 1, one of the two electrodes is fixed on the shaft, the other one is fixed on the cylinder inner wall, and the arc current is brought to the electrodes trough liquid $\mathrm{Hg}$ sliding electrical contacts (6). It is possible to show that the aerodynamic flow conditions that get installed in the rotating cylinder are such that there is no velocity of the air relatively to the electrodes. Moreover, it may be easily shown that the forces acting on the charged particles present in the plasma are several order of magnitude greater than the centrifugal force. We indeed could observe that the plasma was not disturbed even when the vessel was rotated at the maximum frequency. For instance for a same arc current pulse $I(t)$, one obtains the same arc voltage drop $V_{\mathrm{a}}(t)$ for $\omega=0$ and $\omega=300 \mathrm{rad} . \mathrm{s}^{-1}$

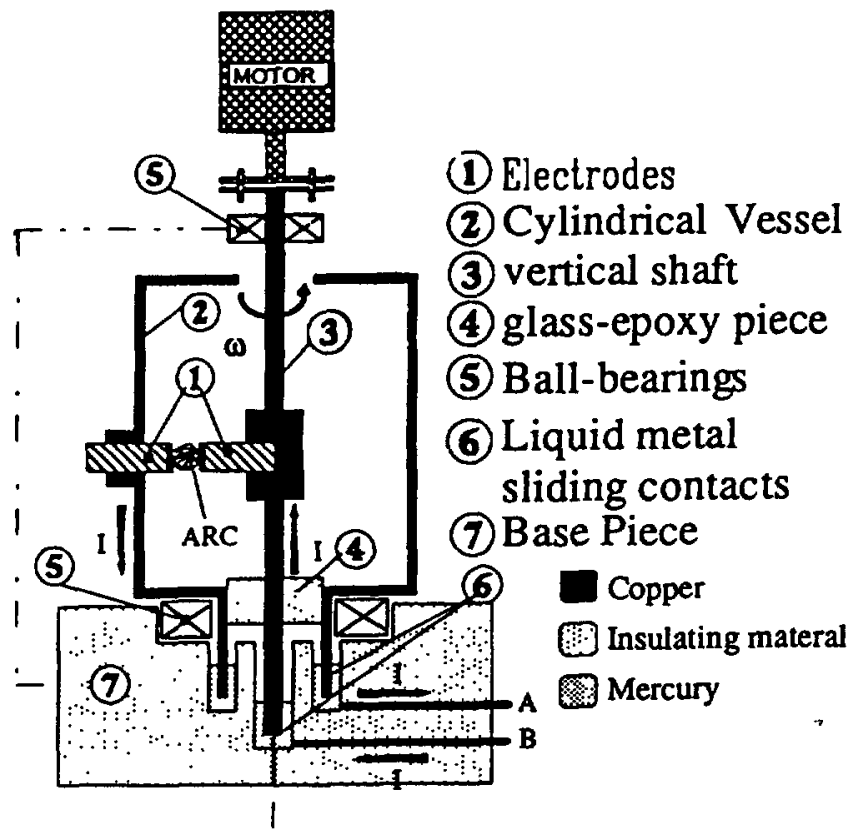

Fig. 1. - Scheme of the mechanical part of the experimental device.

The electrical power supply consists of three parts :

- a main circuit (1) allows to feed the electrodes with a quasi half-sinusoidal current (with 


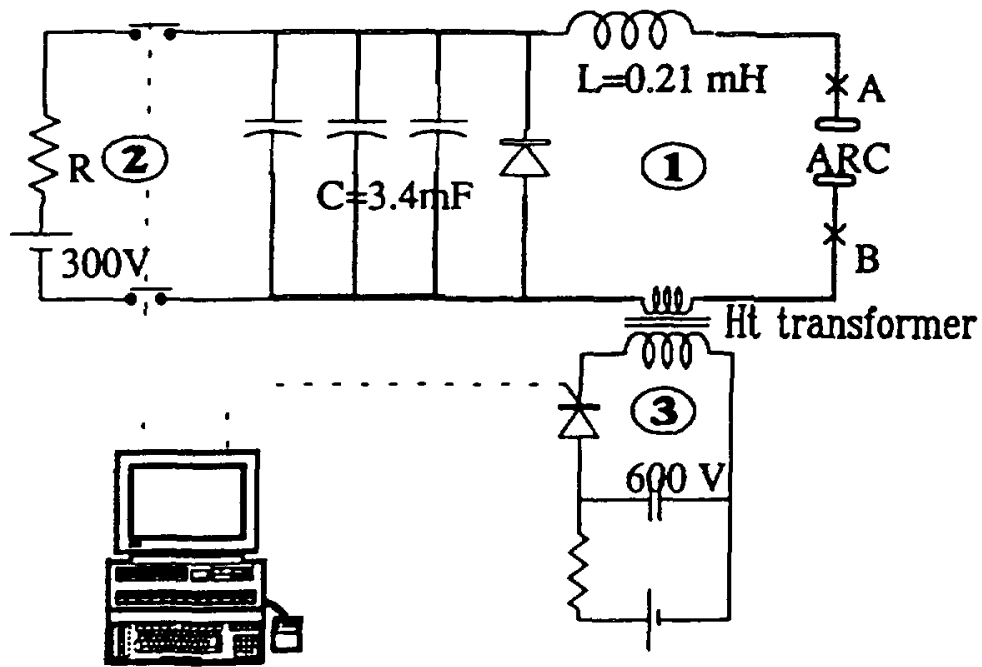

Fig. 2. - Scheme of the electrical part of the experimental setup.

a maximum intensity $I_{\max }$ in the range of 100 to 3000 amperes and a time duration in the range of 1 to 20 milliseconds) ;

- an auxiliary circuit (2) allows to charge the storage capacitor bank up to 300 volts ;

- the last circuit (3) is a trigger circuit. Since a fixed gap of a few millimiters is kept between electrodes, the arc has to be triggered with a high voltage pulse. One should note that the secondary coil of the high voltage transformer must also be able to carry the arc current of a few thousands amperes.

The operating cycle of the experience is controlled with a microcomputer : a great number of successive arcs are necessary, in order to get a mass loss greater than the ordinary precision of our balance $\left(10^{-5} \mathrm{~g}\right)$.

Principle of the experiment. - It is based on a very simple principle : the liquid metal created by the arc on the surface of the "inner " electrode is submitted to a radial acceleration $\gamma=R \omega^{2}$ ( $R$ is the distance to the axis of rotation). When the corresponding centrifugal force becomes sufficient, the whole liquid metal is ejected, and so, this experimental setup allows us to get a direct measurement of the total liquid and vapor quantities by simply weighing the electrodes after a given number of arcs. Moreover, we can assume that the mass transfer from the outer electrode to the inner one is negligible due to the distance of $5 \mathrm{~mm}$ between electrodes and to centrifugal force.

\section{Results and interpretation.}

COMPARISON OF ANODE AND CATHODE EROSION FOR VARIOUS CONTACT MATERIALS. - We have compared the erosion between an anode and a cathode made of two kinds of materials :

- pure silver or pure copper (OFHC) ;

- pseudo-alloys $\mathrm{AgMeO}$ ( $\mathrm{Ag}$ with $\mathrm{MeO}$ grains dispersed inside) such as $\mathrm{AgCdO}$ or $\mathrm{AgSnO}_{2}$.

The various experimental conditions adopted to test these materials are the following :

- the gap between anode and cathode is kept constant and equal to $5 \mathrm{~mm}$;

- the arc current pulse delivered to the electrodes is semi-sinusoidal with the following 
characteristics :

$$
I_{\max } \approx 400 \mathrm{~A} ; \quad \text { Arc duration } \approx 5 \mathrm{~ms} ; \quad \int_{t=0}^{t=5 \mathrm{~ms}} I(t) . \mathrm{d} t \approx 1.4 \mathrm{C} .
$$

- It is important to specify that the experimental mass losses presented were always measured for an electrode (anode or cathode) fixed in an "inner " position (see Fig. 3). Indeed, the " outer » electrode may receive liquid metal droplets ejected from the "inner " electrode surface, and then the measurement of the mass loss for the « outer » electrode would not have any meaning.

The results, presented in figure 4 , correspond to the mass loss per arc versus the acceleration $\gamma$, for the arc current pulse given above.

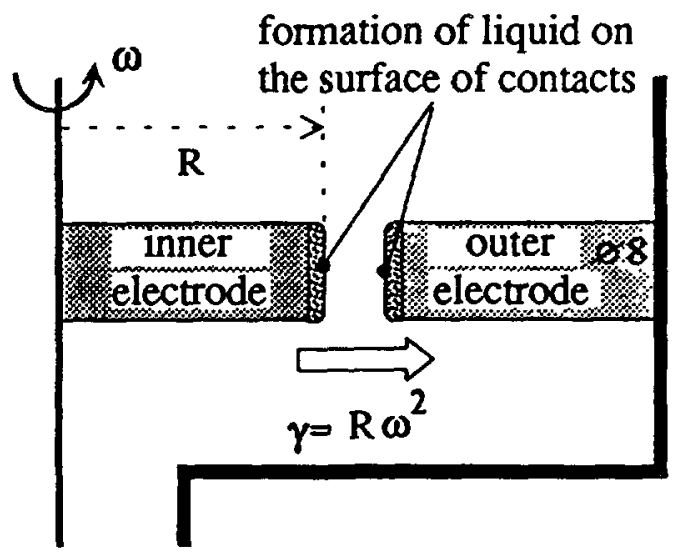

Fig. 3.

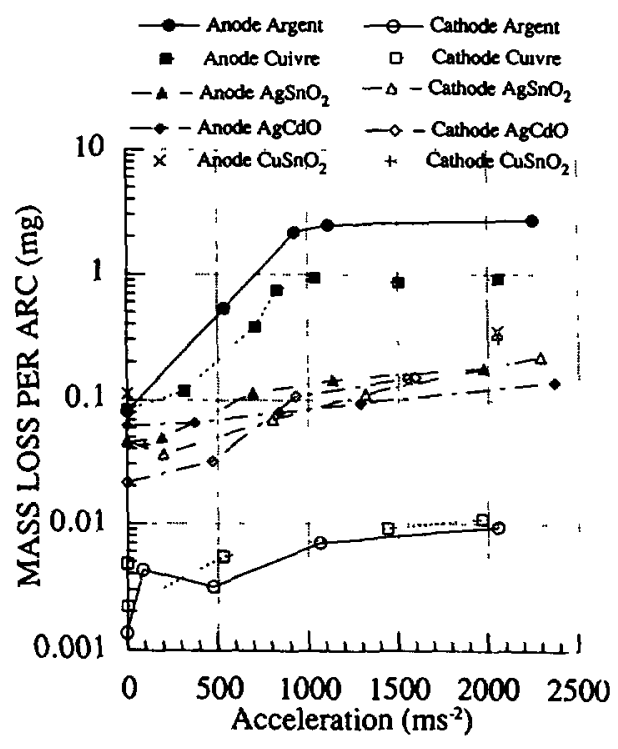

Fig. 4.

Fig. 3. - An illustration of the principle of the measurement of liquid quantities.

Fig. 4. - Comparison of erosion for different contact materials obtained with the experimental rotating setup.

NB : The mass loss is averaged over 500 arcs. We must precise that these results present a very small scattering and that they are independent of the total number of arcs, above a few hundreds ones.

For pure metals such as $\mathrm{Ag}$ or $\mathrm{Cu}$, one can note a great difference of the erosion rate for a same material between the anode and the cathode. For $\mathrm{Ag}$, the anode is nearly 250 times more eroded than the cathode at the maximum value of $\gamma$. This result, which is quite new, is very surprising and shows clearly that there certainly exists a great discrepancy between the heat flux characteristics, applied to the surface of the cathode relatively to the anode.

For the anode, we also observe a very clear saturation effect of the erosion rate above a critical value $\gamma_{c}$ of the acceleration. This result confirms the fact that, above $\gamma_{c}$, the whole liquid metal leaves the surface of the electrode. Moreover, the numerical modelling, that will be described in the next chapter, allowed us to verify that there exists only a little difference 
(30\% maximum) between the liquid quantities calculated with each one of the two following assumptions :

i) the liquid does not leave at all the surface of the electrode (no ejection forces acting on the pool);

ii) the liquid is expulsed from the surface as soon as created by the arc root.

This remark and the results of figure 4, permit to deduce that only a small proportion of the melted zone is ejected when the electrodes are at rest.

Materials containing metallic oxides $\left(\mathrm{AgSnO}_{2}, \mathrm{AgCdO}, \mathrm{CuSnO}_{2}\right)$ present different erosion characteristics : the erosion is quasi similar for both anode and cathode. It takes place at an intermediate level between the anode and the cathode rates of a pure metal. We can also note that the erosion rate versus $\gamma$ does not present a saturation effect as neat as the one shown by pure metals. This may indicate that, for $\mathrm{AgMeO}$ or $\mathrm{CuMeO}$, a certain quantity of the liquid phase can remain on the electrode. In fact, the proportion of oxide (nearly $20 \%$ in vol.) may considerably increase the viscosity of the liquid and also improve its adhesion and wetting properties at the solid-liquid interface. Such a problem is being studied at present time at LGEP.

These results are primordial to help us to understand erosion mechanisms. If, for instance, we consider the case of A.C. switching device electrodes which are alternatively anode or cathode, results of figure 4 (for $\omega=0$ ) lead to the conclusion that for the arc current pulse used in our experiment, pure $\mathrm{Ag}$ contacts will give rise to a similar or even lower erosion rate than AgMeO ones. Of course, such an assertion does not take into account other parameters like electrical contact resistance or static and dynamic welding properties of contacts. If now we do the sum of the total mass loss for the anode and for the cathode as a function of $\omega$, we may see that the mass loss becomes greater for pure metals than for pseudo alloys as $\omega$ increases. Moreover, we have observed that the mass loss is essentially due to the ejection of liquid metal. So the preceding remark may explain why AgMeO are more interesting than pure metals for more severe arc current conditions (for which forces due to the arc itself : Marangoni effect or Lorentz forces, will be able to eject a substantial quantity of liquid).

\section{D modelling of thermal processes occurring in an electrode.}

DESCRIPTION OF THE MODEL. - It would be quite interesting to see whether the preceeding measurements allow to access to a good order of magnitude of the power flux transferred by the arc to the electrode. For that we have elaborated a thermal 2D computer code.

First of all we have to recall some results concerning the arc itself :

- a high speed $16 \mathrm{~mm}$ (5000 fps) laser cinematography [1] showed that, in air at atmospheric pressure, the cathodic arc root as well as the anodic one are working in a concentrated single spot regime and have a circular shape ;

- due to the huge power flux present in the arc root $\left(10^{9}\right.$ to $\left.5 \times 10^{10} \mathrm{~W} / \mathrm{m}^{2}\right)$, the temperature of the material will increase and reach fusion and vaporization temperature. So, this thermal flux transfer problem is a typical ablation problem that involves moving boundaries.

Taking into account the above considerations, we have adopted the following assumptions for the modelling of the thermal phenomena occurring in an electrode submitted to an electric arc :

i) an axisymetric geometry (see Fig. 5) is used ;

ii) a given power density $Q(r, t)$ enters the upper surface of the electrode at its center;

iii) the electrodes are made of a pure metal for which we take into account the various phase changes ; 


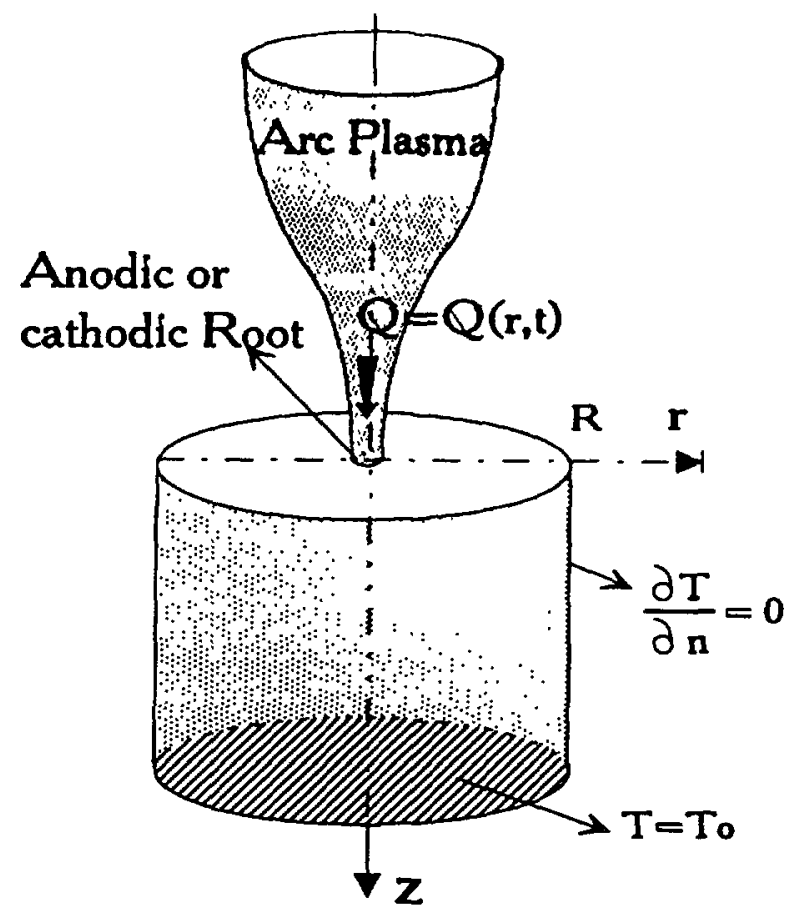

Fig. 5. - The model of arc heat transfer to an electrode adopted in the numerical simulation.

iv) the material is characterized by its constant density $\rho$ (no thermal dilatation) and also by its specific heat capacity $C_{\mathrm{p}}(T)$ and its thermal conductivity $k(T)$, which will be taken both dependent on the temperature $T$;

v) the liquid metal created on the surface remains at rest (no thermoconvection);

vi) the choosen boundary conditions are :

- no heat flux on the lateral sides of the electrode ;

$-T=T_{0}$ fixed on the lower cross section of the electrode.

Mathematical formulation of the problem. - The unstationary equation of heat diffusion has to be solved in the various phases (solid and liquid), after suppression of the terms of thermoconvection and viscous dissipation. Moreover, heat balance equations across phase boundaries (solid-liquid and liquid-vapor) have also to be taken into account.

Our approach consisted in the use of an enthalpy function $H(T)$ permitting to replace this complicated set of equations by a much simpler one that may be written :

$$
\rho \frac{\partial H(T)}{\partial t}=\operatorname{div}(K(T) \cdot \bar{\nabla} T)+S
$$

$S$ is a body source term (such as Joule Effect).

A piecewise linearization (see Fig. 6) allowed us to suppress the discontinuities of $H$ function for fusion or vaporization.

Such a method has already been described by several authors [2]. It permits to avoid taking into account the location of phase change boundaries at each time step of the calculation of the temperature field $T(r, z, t)$. These boundaries may be determined only after the calculation of $T$ has been achieved. It is, of course, necessary to verify that the result does not depend on the temperature interval $T_{2}-T_{1}$ (see Fig. 6). 


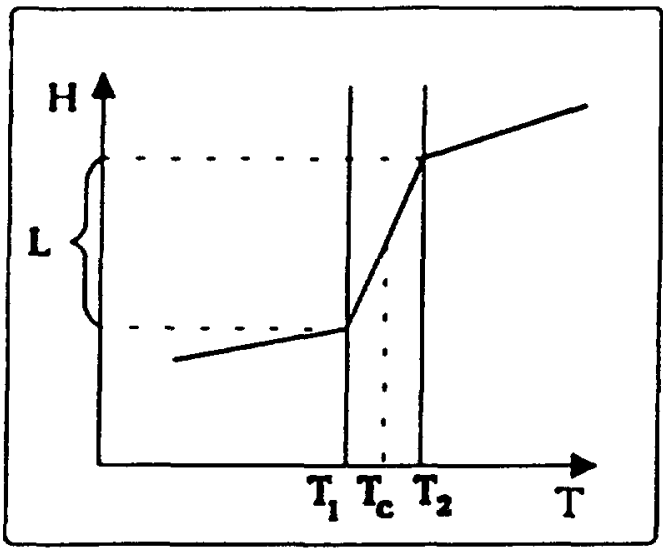

Fig. 6. - Linearization of the enthalpy function $H(T)$.

The problem of ablation. - As soon as vaporization occurs, the surface on which the heat flux is applied does not remain the same. In order to solve the numerical difficulties related to this particular moving boundary, we propose an original method that consists in replacing the vaporized zone with a fictitious one (see Fig. 7) that shall be governed by the following equation : $\mathrm{d}^{2} T / \mathrm{d} z^{2}=0$ and in applying a fictitious power flux $Q_{\mathrm{s}}(r, t)$ on the upper surface of the grid. The physical imposed power flux that we wish to apply on the liquid-vapor interface is $Q(s, t)$, where $s$ is the curvilinear coordinate. So, as soon as vaporization occurs, a correction has to be made (at each time step) in order to determine $Q_{s}(r, t)$ allowing to ensure the desired value of $Q(s, t)$.

The Kirchhoff transformation. - It is important to note that, for metals like $\mathrm{Ag}$ or $\mathrm{Cu}$, the conductivity in the liquid is very different from that in the solid state.

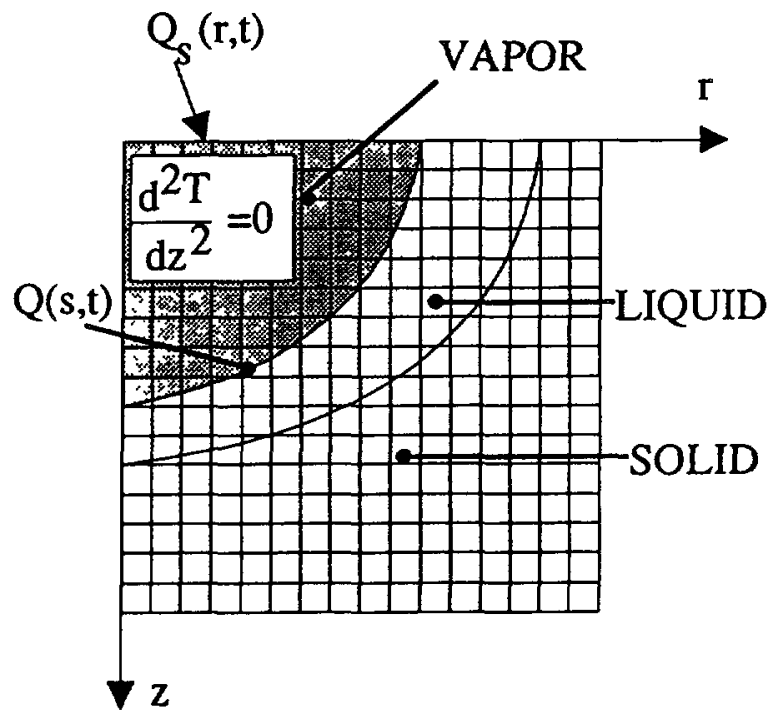

Fig. 7. - Modelling of vapor ablation. 
The well-known Kirchhoff transformation [3] allows, by a simple coordinate change to take these variations of thermal conductivity into account.

Numerical treatment of the equations. - We used a finite difference method and the equations were numerized by using an implicit scheme of discretization. Then a linear system of equations was obtained and solved with a SOR (Successive Over Relaxation) type algorithm.

The validation of this computer code was made with analytical or numerical results issued from literature.

Now, we shall present the influence of the power density distribution $Q(s, t)$ on the liquid or vapor quantities. For that, a fixed power $P$ and two uniform power densities $Q_{1}$ and $Q_{2}$ were considered. In figures $8 \mathrm{a}$ and $8 \mathrm{~b}$, we give for a $8 \mathrm{~mm}$ diameter copper electrode, the calculation results in two cases :

$P=5000 \mathrm{~W}$ and $Q=2 \times 10^{9} \mathrm{~W} / \mathrm{m}^{2}$, the isotherms being given at time $t=1.5 \mathrm{~ms}$.

$P=5000 \mathrm{~W}$ and $Q=2 \times 10^{11} \mathrm{~W} / \mathrm{m}^{2}$, the isotherms being given at a much shorter time $t=40 \mu \mathrm{s}$.

$N B:$ In the graphs presented in figures $8 \mathrm{a}$ and $8 \mathrm{~b}$, the heat source is applied at the lower left hand corner (points 1 to 20).

We note that in the first case, for a stagnation time of $1.5 \mathrm{~ms}$ no metal has been vaporized, whereas in the second case, a rather deep and narrow hole has been made after a much smaller stagnation time.

Those examples of calculation show very clearly the influence that the power density may have on phase changes.
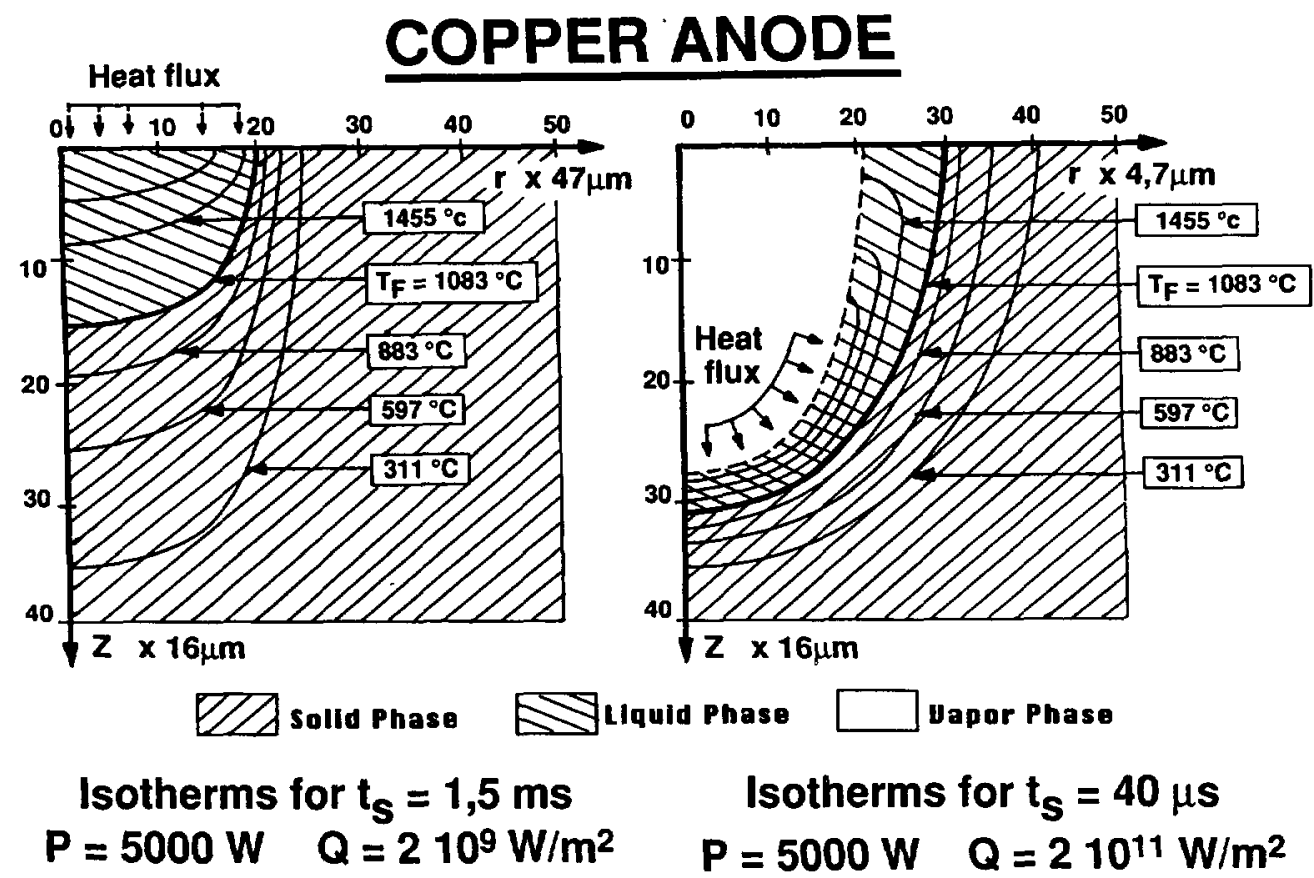

a)

b)

Fig. 8. - a) Isotherms at time $t=1.5 \mathrm{~ms}$ for $P=5000 \mathrm{~W}, Q_{1}=2 \times 10^{9} \mathrm{~W} / \mathrm{m}^{2}$. Material is pure copper. b) Isotherms at time $t=40 \mathrm{~ms}$ for $P=5000 \mathrm{~W}, Q_{2}=2 \times 10^{11} \mathrm{~W} / \mathrm{m}^{2}$. Material is pure copper. 
That thermal computer code is a very useful tool that we may use for the determination of the evolution of fusion and vaporization boundaries as a function of time. We also think that, by comparing the preceding experimental measurements of the liquid quantity to the calculation results (with various values of the arc current intensity $I(t)$ or of the electrode radius), it will be possible to determine $Q(s, t)$. For such a purpose, a few hypothesis have to be made on the form of $Q(s, t)$. At the present time a study has been undertaken at LGEP that will take into account the physical phenomena in anodic or cathodic arc roots.

\section{Determination of the characteristics of the power flux applied on a copper anode.}

We have seen that our experimental device allowed us to determine the quantity of liquid and vapor created by the arc on an electrode. This has been done for a given current pulse having a quasi semi-sinusoidal form : $I(t)=I_{\max } \sin (\pi t / T)$ for $0<t<T$ and $I(t)=0$ for $t<0$ or $t>T$. For $T$ equal to 5 milliseconds and $I_{\max }$ ranging from 400 to $1200 \mathrm{~A}$, we measured the mass loss per arc versus $I_{\max }$ for a $8 \mathrm{~mm}$ diameter copper anode at the maximum value of $\omega$.

In a first time we have tried to see whether a very simple expression for $Q(s, t)$ allowed us to approach the experimental results. For that we supposed that the heating of the anode was only due to the incident electron flux as it was explained in [4]. Then if we take a constant and uniform current density $J_{0}, Q(s, t)$ has got the following form :

$$
\begin{aligned}
& Q(s, t)=J_{0} \cdot V \text { for } 0 \leqslant s \leqslant a=\sqrt{I(t) / \pi J_{0}} \\
& Q(s, t)=0 \text { elsewhere. }
\end{aligned}
$$

The voltage $V$ is connected to the anodic voltage fall $V_{\mathrm{a}}$ by the relation $V=V_{\mathrm{a}}-W_{\mathrm{f}} / q$ in which $W_{\mathrm{f}}$ is the Fermi level in $\mathrm{eV}$ of the considered metal and $q$ the electronic charge. $a$ is the radius of the anodic root and $I(t)$ the arc current intensity.

Our thermal model allowed us to calculate the liquid and vapor quantities for various values of $J_{0}$ and $V_{\mathrm{a}}$ (copper anode). The results are presented in figure 9 for a fixed value $J_{0}$ and different values of $V$ between 2 and 10 volts. The value $J_{0}=2 \times 10^{8} \mathrm{~A} / \mathrm{m}^{2}$ does not result from a random choice. It is issued from previous current density measurements made with a force measurement device [5]. For such a value of the anodic current density, we can note (see Fig. 9) that the ablation mode choosen for the calculations (a) the liquid does not leave at all the surface of the electrode, or $b$ ) the liquid is expulsed as soon as created by the arc root) has little influence on the total amount of liquid + vapor.

In figure 9 we have also presented the experimental erosion curve. We immediatly may remark that an expression for $Q(s, t)$; as simple as the one proposed above allows to get the value of $V_{\mathrm{a}}$ that is necessary to obtain the same total quantity of liquid and vapor as the one obtained for a copper anode with the experimental rotating device.

\section{Conclusion of the study.}

An original experimental setup allowed us to reach, for the first time, a good evaluation of the total liquid and vapor quantities created by an arc on an electrode.

The achievement of a bidimensional numerical thermal modelisation permitted us to calculate the time evolution of the fusion and vaporization boundaries for a pure metal electrode submitted to a power density $Q(s, t)$ and, thus, to evaluate the quantities of liquid and vapor created.

The comparison of experimental results and of calculation results allows then to reach a good approximation of the power flux impinging the anode or the cathode. At present, further studies have been undertaken in order to obtain a better characterization of these power fluxes. 
Erosion curve as a function of

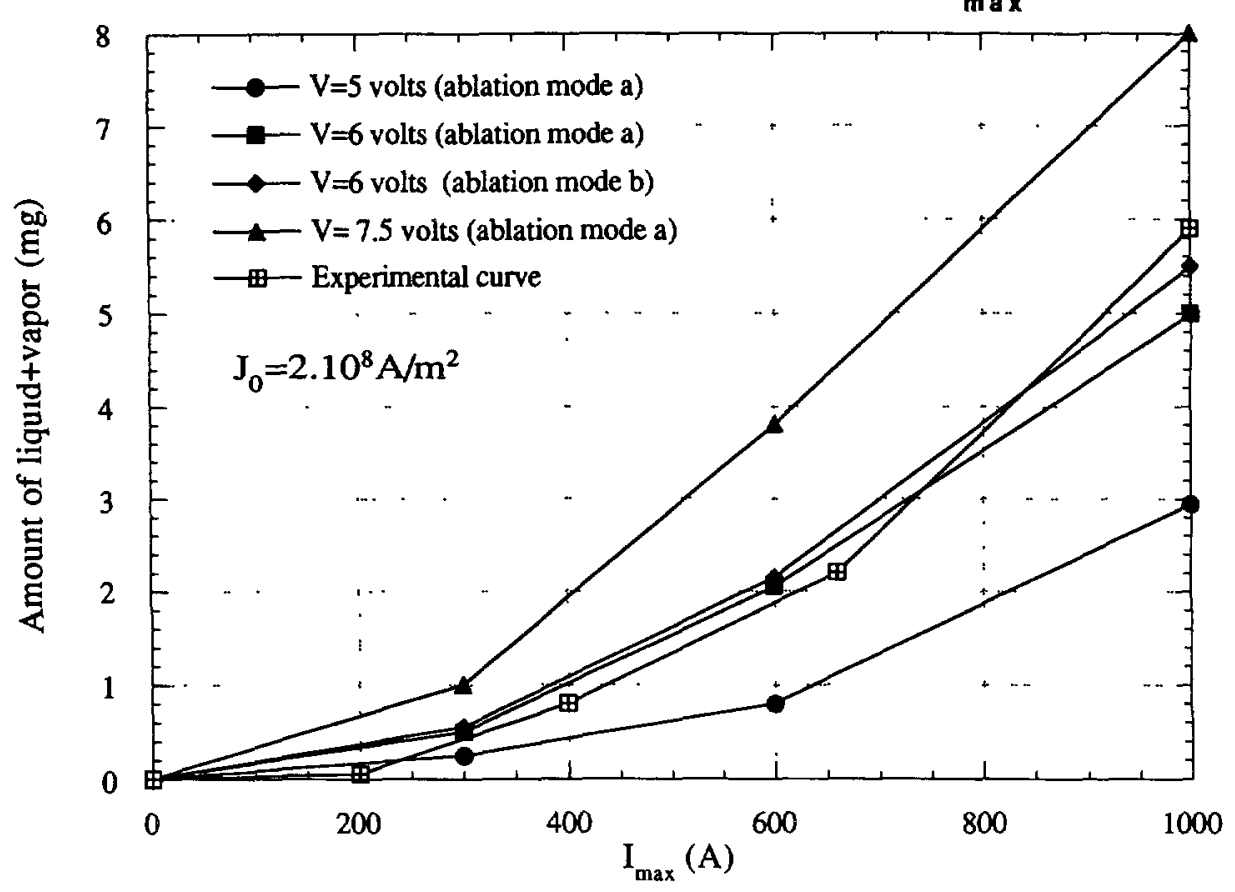

Fig. 9. - Total quantity of liquid and vapor created on a copper anode for a semi-sinusoidal arc current pulse of maximal intensity $I_{\max }$.

\section{References}

[1] Chabrerie J. P., Devautour J., Contribution to the study of interactions between an atmospheric pressure arc root and $\mathrm{Cu}, \mathrm{Ag}$ or $\mathrm{AgMeO}$ electrodes (ICEC, Montreal 1990).

[2] Hunter L. W., Kutter J. R., The Enthalpy Method for Heat Conduction Problems with moving boundaries. J. Heat Transfer 111 (May 1989).

[3] Kirchhoff G., Vorlesungen über der theorie der Warme (Barth, Leipzig, 1894).

[4] Pfender E., Pure Appl. Chem. 48 (1976) 199-213.

[5] Chabrerie J. P., Haidar J., Experimental Study of forces acting on arc electrodes. Proceedings of the 14th Int. Conf. on Elect. Contacts (Paris, juin 1988). 